C 2016 IEEE. Personal use of this material is permitted. Permission from IEEE must be obtained for all other uses, in any current or future media, including reprinting/republishing this material for advertising or promotional purposes, creating new collective works, for resale or redistribution to servers or lists, or reuse of any copyrighted component of this work in other works.

Digital Object Identifier 10.1109/ECCE.2016.7855513

IEEE Energy Conversion Congress and Exposition (ECCE), Milwaukee, WI, USA

Thermal-based finite control set model predictive control for IGBT power electronic converters Johannes Falck

Markus Andresen

Marco Liserre

Suggested Citation

J. Falck, M. Andresen and M. Liserre, "Thermal-based finite control set model predictive control for IGBT power electronic converters," 2016 IEEE Energy Conversion Congress and Exposition (ECCE), Milwaukee, WI, 2016, pp. 1-7. 


\title{
Thermal-based Finite Control Set Model Predictive Control for IGBT Power Electronic Converters
}

\author{
Johannes Falck, Student Member, IEEE, Markus Andresen, Student Member, IEEE, Marco Liserre, Fellow, IEEE \\ Chair of Power Electronics, Christian-Albrechts-Universität zu Kiel, Kiel, Germany \\ Email: jofa@tf.uni-kiel.de,ma@tf.uni-kiel.de,ml@tf.uni-kiel.de
}

\begin{abstract}
Thermal cycling is one of the main sources of aging and failure in power electronics. A possibility to reduce the thermal stress of semiconductors is to control the losses occurring in the semiconductor devices with the target to reduce the thermal cycles. This approach is known as active thermal control. The hardest limit of the existing active thermal control approaches is that they do not offer a general framework where the optimal switching sequence is selected in order to fulfill the applications demand and reduce the thermal stress in specific semiconductors. The goal is to achieve the minimum thermal stress for the best possible overall performance. For this purpose finite control-set model predictive control (FCS-MPC) seems the optimal approach because it allows including of non-linear thermal and lifetime related models into the control law. A precise control of the thermal stress in the semiconductors can be achieved as the optimal switching vector is directly applied to the physical system. This allows to avoid overrating of the used module or to increase its lifetime. In the paper the approach is proven using simulation and experimental results.
\end{abstract}

Index Terms-Power Electronics Reliability, Active Thermal Control, Junction Temperature Estimation, Lifetime Prediction

\section{INTRODUCTION}

Power semiconductors are increasingly used not only in traditional fields like variable speed drives, consumable electronics and in new but consolidated ones like renewable energies but also in emerging fields like more-electric aircrafts and medical systems. Presently, the control system technology finds itself in a paradigm-changing tipping point, in which more demanding control goals, system flexibility, and functionalities required by emerging applications are driving the control system technology development, in addition to stabilization and robustness, which was the main focus in the past [1]. The reliable operation is becoming crucial for the safety of several key areas like energy, health and transportation [2]. To reduce the required system size, material consumption and working time, power semiconductors in the lower and medium power range are often assembled into modules [3]. An important cause of aging and failures of these modules is the cyclic heating and cooling processes, so called thermal cycles [4]. Manufacturers face this problem by improving the assembly and materials which suffer from mechanical fatigue [5]. These solutions attempt at reducing the effects of thermal cycling without curing the cause of the problem.

Only limited research has been carried out to reduce the thermal cycling with active thermal control, which aims at smoothing the fluctuation of the junction temperature of the semiconductors during operation. Chosen control variables are the switching frequency [6], the modulation method [7], the dc link voltage [8], reactive circulating current [9], [10] and active circulating current [11]. In these approaches promising results could be achieved that motivate for further research in active thermal control. However, the main disadvantage of active thermal control is the decrease of the overall system efficiency or system performance [12].

The drawback of the cited control approaches is that they do not offer a general framework where the optimal switching sequence is selected in order to fulfill the applications demand and to reduce the thermal stress in a specific semiconductor while limiting the additional losses. A second limitation is found in the limited availability of junction temperature measurement or estimation methods needed to implement the control. Also aging and lifetime consumption of the module are not yet taken into account in active thermal control approaches. More open topics in research are found in giving design guidelines for temperature control and providing an universal approach that is not restricted to a specific packaging and semiconductor technology.

This paper presents a control approach that aims at overcoming these limitations to widen the use of active thermal control in power electronic applications. For this purpose finite control-set model predictive control (FCS-MPC) seems the optimal approach because it allows an optimal control of every switching event and including of non-linear thermal and lifetime related models into the control law. A precise control of the thermal stress in the semiconductors can be achieved as the optimal switching vector is directly applied to the physical system. A model for online junction temperature estimation that is suited for the use of FSC-MPC is designed.

In the following the influence of the temperature cycles on power electronic modules is described in section II. The active thermal control design is performed in section III and the online temperature estimation technique adapted to FCSMPC is presented in section IV. Simulation results are given in section $\mathrm{V}$ and an experimental validation using a high speed infrared camera is shown in section VI. Finally the conclusion is given in section VII.

\section{INFLUENCE OF THE JUNCTION TEMPERATURE IN POWER CONVERTERS}

In this section the influence of the junction temperature on power electronic modules is described first and afterward 


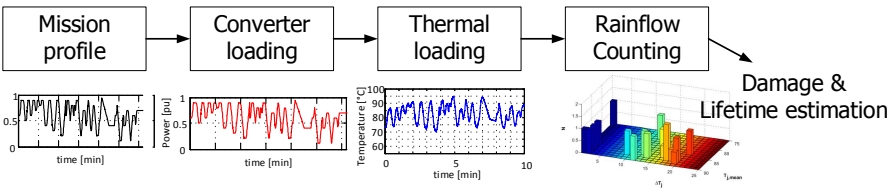

Fig. 1. Procedure to transfer the mission profile into the thermal loading of the devices and to obtain a lifetime estimation.

the particular problem in the application for electric drives is explained.

\section{A. Junction temperature modeling and lifetime estimation}

Most failures of power electronic modules related to wear out are caused by the temperature fluctuations on the chip [13]. The stress in the module occurs at the interconnections of layers consisting of materials with different coefficients of thermal expansion. These different layers are required to ensure good heat dissipation due to the losses of the power semiconductors and the requirement for electrical isolation among the chips and the module's baseplate. Fluctuations of the temperature and temperature gradients cause mechanical stress between these layers, which leads to wear out and finally failure of the component. As a consequence, the most dominant failure mechanisms are related to the junction temperature in the direct bonded copper (DBC) structure, such as chip solder fatigue, bond wire lift off and baseplate solder fatigue [13].

Based on the influence of the junction temperature variation, manufacturers commonly express the lifetime of their power semiconductors in number of thermal cycles to failure $N_{f}$ with the magnitude $\Delta T$ and the influence of the average junction temperature $T_{j, \text { mean }}$ as shown in (1).

$$
N_{f}=a_{1} \cdot\left(\Delta T_{j}\right)^{-a_{2}} \cdot e^{-a_{3} T_{j, m e a n}}
$$

In this equation, the constants $a_{1}, a_{2}$ and $a_{3}$ are empirically obtained. However, $a_{2} \approx 5$, which makes the thermal cycles a major influence on the lifetime. Consequently, for an estimation of the lifetime, the knowledge of the junction temperature is important. The junction temperature is commonly expressed by a convolution integral of the power semiconductor losses $P_{\text {loss }}$ with a thermal impedance. For the thermal impedance from junction to case $Z_{t h, j c}$, which is usually provided by the manufacturer, this is expressed in (2) with the case temperature $T_{\text {case. }}$.

$$
T_{j}(t)=Z_{t h, j c}(t) * P_{l o s s}(t)+T_{\text {case }}(t)
$$

The procedure to utilize the junction temperature for an estimation of the lifetime is shown in Fig. 1. With the knowledge of the mission profile, the converter loading is obtained, which is translated with the information about the electrical and thermal system into a junction temperature profile. From the junction temperature profile in the time domain, the thermal cycles are commonly counted with the rainflow counting algorithm [14]. Finally, to obtain a lifetime estimation, the counted thermal cycles are evaluated in a linear extrapolation in the lifetime model expressed with (1). It needs to be pointed out, that by applying rainflow counting algorithm, the information about the time period of the thermal cycles is lost.

\section{B. Junction temperature impact in electric drives}

In many power converters applications, the thermal cycles induced by the mission profile are the most relevant ones. Examples are $\mathrm{dc} / \mathrm{dc}$ converters or grid connected applications, where the fundamental output frequency of the current is $50 / 60 \mathrm{~Hz}$ or higher. In this case the influence of the thermal cycling in the fundamental frequency is commonly neglected.

However, in power converters for electric drives, the fundamental frequency is varying and can result in conditions in which the thermal cycling in the fundamental period is limiting the lifetime of the converter. During low speed and high torque of drives, the power semiconductors undergo thermal cycles with high magnitude [15]. In order to overcome this, overrating of the components has been a solution. This brings additional costs in the system design, because the design has to be made for the worst case conditions of the mission profile. Another solution, to reduce the overrating, is the industrially invented concept of active thermal control [15]. During conditions with high torque and low speed, the converter is reducing the switching frequency and regulating the output current for preventing excessive thermal cycling [16].

\section{ACTIVE THERMAL CONTROL DESIGN}

Active thermal control methods smooth the junction temperature progression in the module's semiconductors during operation. Their goal is to to keep the thermal swing as small as possible [17]. As a consequence thermo-mechanical stress is released from the semiconductor chips which leads to increased lifetime of the module [5], [18]. Additional losses may occur in the module in order to raise the temperature temporary. Moreover the total harmonic distortion (THD) may raise due to omitting of switching operations [19] in order to lower the temperature temporary. These negative influences of active thermal control may be considered as inappropriately high compared to the achieved reduction of thermal cycling for particular operating points. This is the main drawback of active thermal control.

\section{A. Optimization aspects}

To decide whether the reduction of a thermal cycle is reasonable in a particular situation during converter operation, an optimization has to be done. The task of the optimizer is to considers both, advantages and drawbacks of usage of active thermal control to come to a decision whether an emerging thermal cycle should be compensated or not. This optimization needs to be done in every switching instant in order to react on high derivatives in the junction temperatures.

The main aspects of the optimization are to fulfill the application demand (in current reference and THD), to reduce the thermal stress and to maintain the the full system performance. 


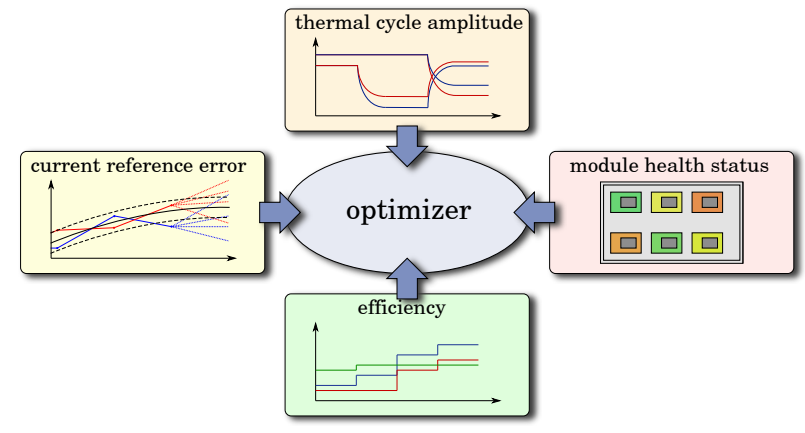

Fig. 2. Main optimization goals of thermal-based FCS-MPC.

In future other aspects may be added like the module health status. This means the stress in a specific semiconductor can be released in order to prevent its specific failure and therefore the failure of the module. The optimizer is shown in Fig. 2.

\section{B. Finite control set model predictive control}

Predictive control has been proposed in power electronics systems for more than 30 years [20], [21]. Its strength are demanding control goals that require non-linear models or multiple control variables [1].

The main principle of MPC is to use a model of the system to predict its dynamic behaviour during operation. The system outputs are computed by evaluation of a cost function on the basis of this prediction. Fig. 3 shows the general structure of MPC. The main parts are the system model, the cost function and the control law which is derived from the optimizer [22]. The system model holds electrical and thermal information of the converter, that are necessary to predict current and temperature progression. The calculation of the system outputs is done using the receding horizon principle. To this, the optimal future output is evaluated each sample and subsequent it is applied to the system. In the next sample this calculation is repeated using updated measurement inputs. This ensures a feedback, making MPC a type of closed-loop control [23].

Switching elements in power electronic systems can be driven either by using a modulator or the switching signals are generated directly by the controller. As only a finite amount of switching states is possible in power electronic inverters, the latter can be realized using FCS-MPC. This is advantageous as no modulator is needed and every switching operation can be determined by the cost function of the control algorithm. As a consequence the switching frequency is variable.

Assuming a three-phase two-level voltage source inverter (VSI) topology, as it is used in a variety of applications in electric drives or grid injection, a total amount of $2^{3}=8$ switching states is possible. From these valid switching states, six are active and two are zero vectors. In FCS-MPC, the model is used to predict the consequences of each possible switching operation to the depth of the prediction horizon. The cost function is used to evaluate which switching operation is optimal and it is then applied to the physical system.

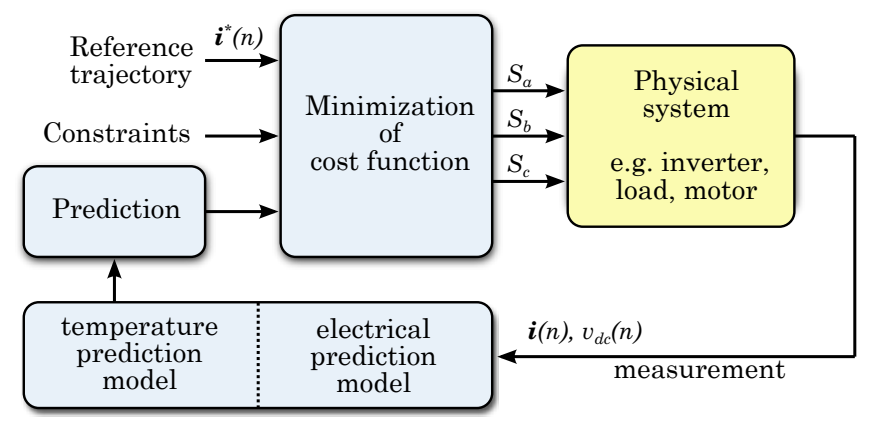

Fig. 3. Control Structure of thermal-based FCS-MPC.

The FCS-MPC allows to predict and evaluate the advantages and drawbacks of the possible thermal control options online. As a result, thermal stress is reduced only when it is reasonable. At the same time unnecessary losses or deviations from the application reference are prevented.

\section{Establishment of a cost function}

The cost function that needs to be minimized consists of a compilation of all quantities that are involved in the optimization and the constraints of the system. Therefore, it includes the current reference error, the amount of thermal swing and the amount of energy that is necessary for the predicted switching operation. As a constraint the maximum junction temperature is added next to the electrical maximum ratings of the system. The cost function is established in the following for each part in particular.

First part is the current reference error. Typically the absolute value of current error to its reference value is chosen for both, $\alpha$ and $\beta$ component of the current [24]. The cost function for the current error $g_{i}$ for sample time instant $n$ and space vector $k$ is denoted as:

$$
g_{i}(n, k)=\lambda_{i} \cdot\left(\left|i_{\alpha}^{*}-i_{\alpha, k}\right|+\left|i_{\beta}^{*}-i_{\beta, k}\right|\right)
$$

In this equation $i_{\alpha}^{*}$ and $i_{\beta}^{*}$ are the current reference values. $i_{\alpha, k}$ and $i_{\beta, k}$ are the predicted currents for the applied space vector $k$. The current reference is assumed to be constant for sampling instant $n$.

Next, the junction temperatures $T_{j, k}^{l}$ for each semiconductor $l$ in the module are considered for each space vector $k$. To detect thermal swings, the deviation from the junction temperatures average value $\overline{T_{j}^{l}}$ are computed. The average is created using a low-pass filter with a time constant adjusted to maximum length of a thermal cycle that has to be reduced by the thermal controller (i.e. $\tau=30 \mathrm{~s}$ ). Each term is weighted with $\lambda_{T^{l}}$.

$$
g_{T_{j}^{l}}(n, k, l)=\lambda_{T^{l}} \cdot\left|\overline{T_{j}^{l}}-T_{j, k}^{l}\right|
$$

Different values for $\lambda_{T^{l}}$ can be used to quantify the stress that should be relieved from semiconductor $l$. If it is equal for each chip, the dependency from $l$ can be removed by using the average of all $q$ chips on the module: 


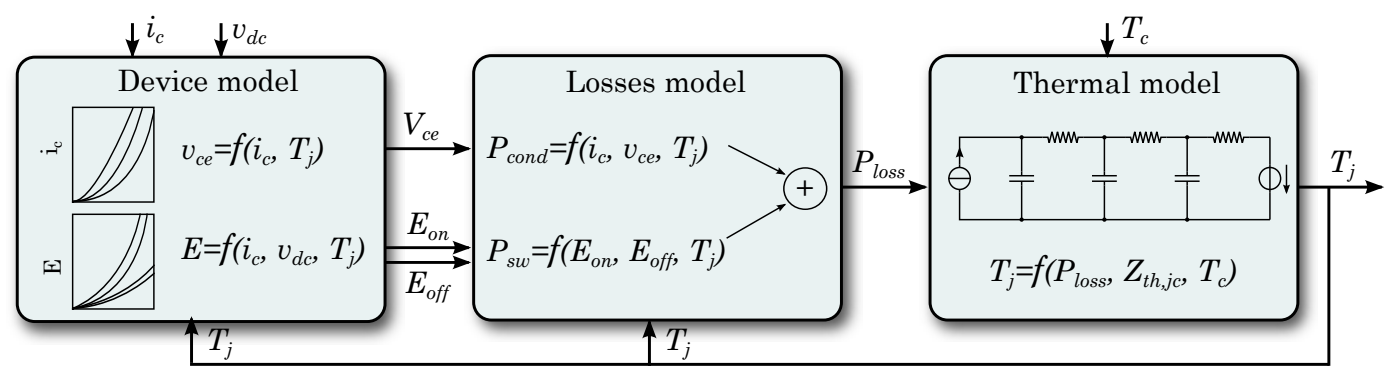

Fig. 4. Online junction temperature estimation model for FCS-MPC.

$$
g_{T_{j}}(n, k)=\frac{1}{q} \sum_{l=1}^{q} g_{T_{j}^{l}}(n, k, l)
$$

The third part of the cost function includes the efficiency, which means reduction of the occurring losses. The dominating losses in the module are the conduction losses and the switching losses [3]. The switching losses can be reduced by choosing space vectors that are neighbored to the previous vector as less semiconductors have to change conductivity [25]. Also avoiding of switching can be preferred if it complies with the current reference tolerance.

$$
g_{P_{\text {loss }}}(n, k)=\lambda_{P_{\text {loss }}} \cdot \sum_{l=1}^{q}\left(P_{s w, k}^{l}+P_{c o n d, k}^{l}\right)
$$

The calculation of the switching losses and conduction losses is done in the junction temperature estimation procedure. These values are reused for the cost function optimization.

\section{JUCTION TEMPERATURE ESTIMATION}

To detect thermal cycles the online knowledge of the junction temperatures are essential. For the given control approach also prediction of the temperatures is necessary. Model-based estimations using a thermal network of the used module and an online calculation of the power losses are known to PWM based control systems as done in [26]. To be used in a direct control structure like FCS-MPC it needs to be adapted.

However, as the selection and direct application of space vectors is a core element of FCS-MPC, it is convenient to calculate the arising energy loss. This is done based on datasheet parameters of the used module and measurements of the load current and the dc voltage as shown in Fig. 4. Afterward a thermal network is used to estimate the junction temperatures.

\section{A. Losses calculation}

The conduction losses of an IGBT $P_{\text {cond,igbt }}$ are computed in dependency of the collector current $i_{c}$ and the collectoremitter voltage $v_{c e}$. This voltage drop is substantially dependent on $i_{c}$, the junction temperature $T_{j, i g b t}$ and the applied gate voltage $v_{g e}$.

$$
P_{\text {cond }, i g b t}=i_{c} \cdot v_{c e}\left(i_{c}, T_{j, i g b t}, v_{g e}\right)
$$

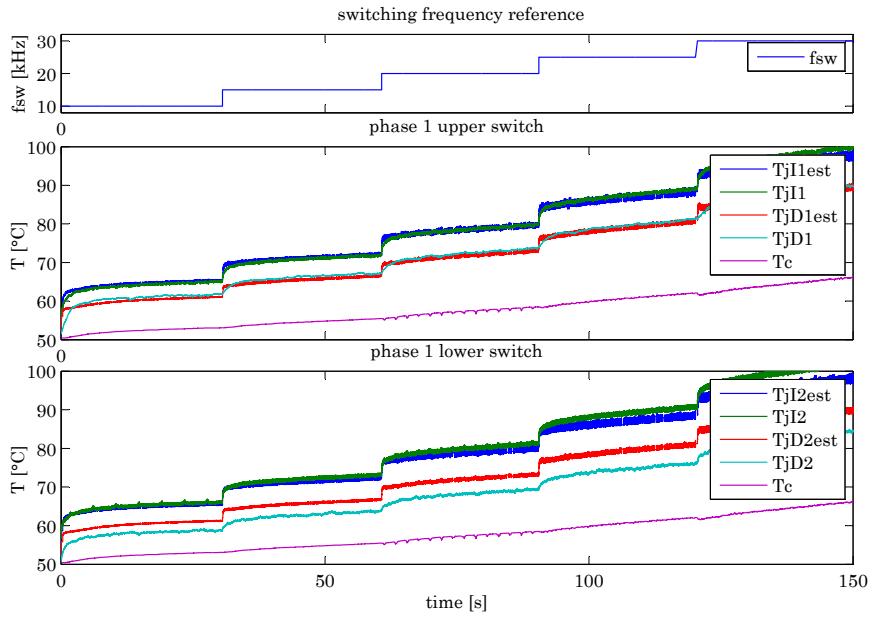

Fig. 5. Experimental validation of the junction temperature estimation. Various switching frequencies are used to show the performance for different ratios of switching losses and conduction losses. Measurement done using a high speed infrared camera, dc voltage $v_{d c}=400 \mathrm{~V}$.

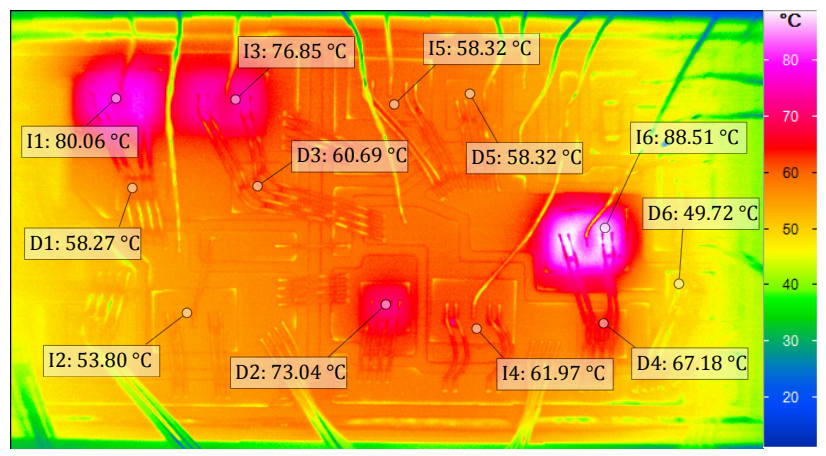

Fig. 6. Infrared camera snapshot of an opened IGBT module during operation.

Conduction losses in power diodes $P_{\text {cond,diode }}$ have a similar structure. They are depend on the forward current $i_{f}$ and its forward voltage $v_{f}$ characteristic, which is dependent on $i_{f}$ and the junction temperature $T_{j, \text { diode }}$.

$$
P_{\text {cond,diode }}=i_{f} \cdot v_{f}\left(i_{f}, T_{j, \text { diode }}\right)
$$

The characteristics of the chips are placed in look-up tables for online computation. As usually a large range of linear behavior is present, the look-up tables can be written compact. 

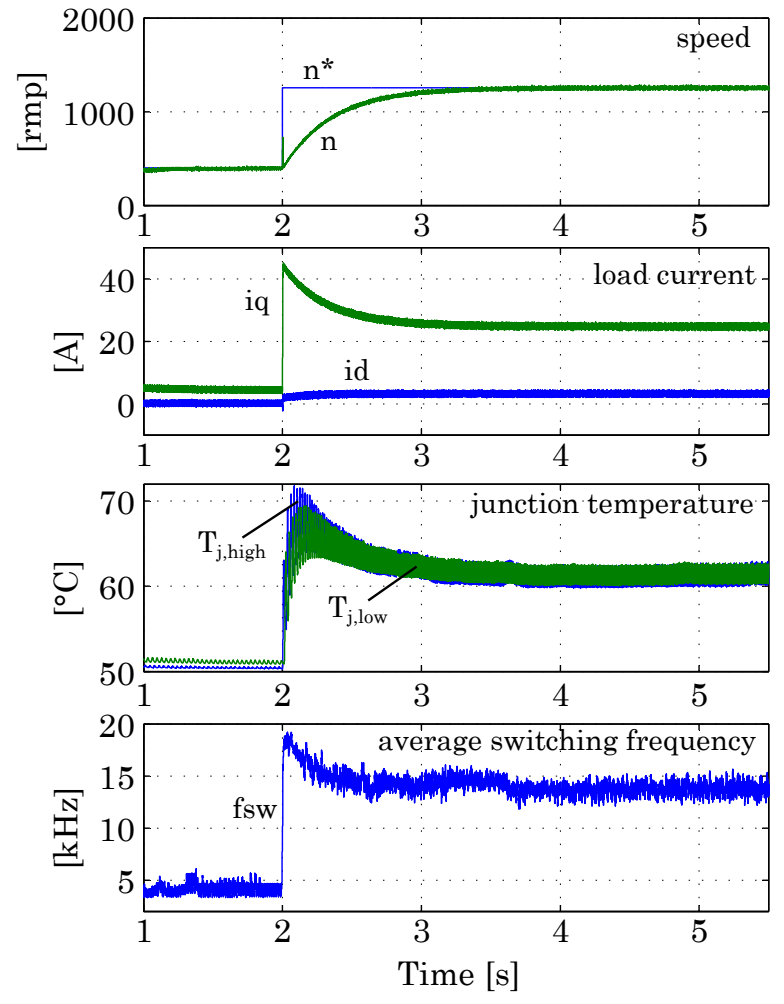
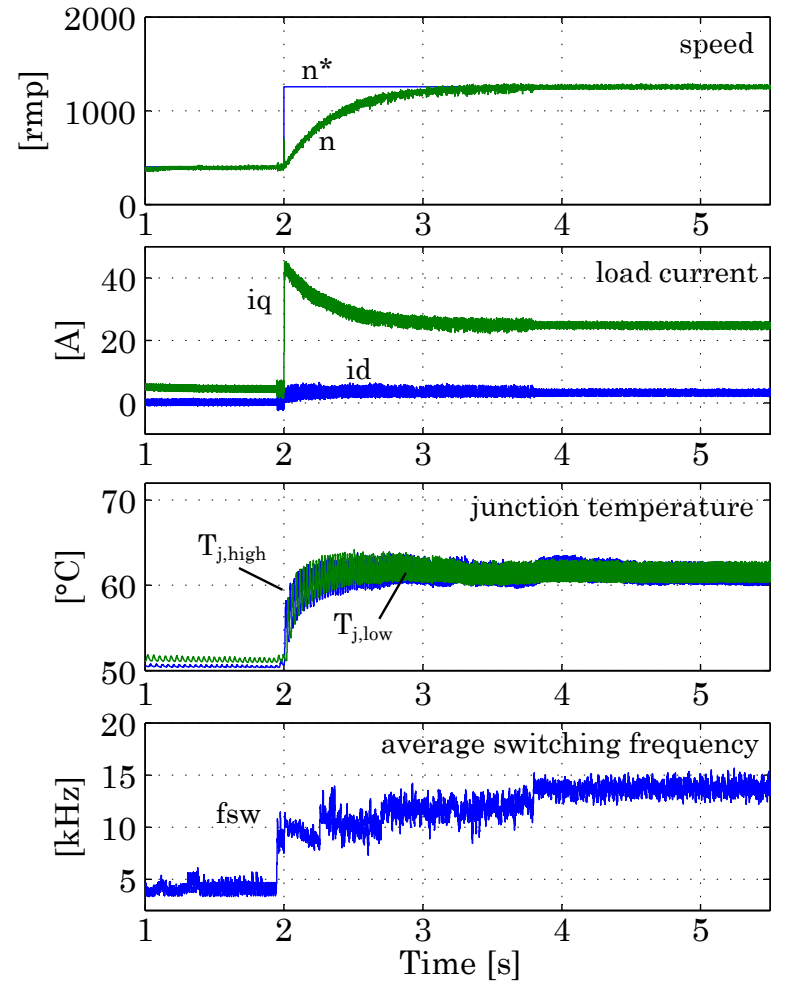

Fig. 7. Simulation of a PMSM. Conventional FCS-MPC (left) and thermal optimized FCS-MPC (right).

The amount of switching losses in the chips depends on the switching energies $E_{o n}$ and $E_{o f f}$, which depend mainly on $i_{c}$, $v_{c e}$ and $T_{j}$. As the FCS-MPC selects a specific space vector in every sampling instant, the switched elements in the module can be identified by a simple comparison with the previous applied space vector. Therefore, each semiconductor obtains either one of the given switching energies $E_{\text {on }}$ and $E_{\text {off }}$ or 0 if no commutation happened in the regarded semiconductor.

\section{B. Thermal model}

A linear Cauer-type thermal network is used to describe the heat propagation in the power electronic system. Parameter values for the thermal resistances and capacities are given by the module's datasheet. A complete estimation of the temperatures from ambient to the junctions needs high computational effort. The temperature estimator can be simplified and its performances improved when taking into account the use of low-bandwidth measurement of the modules case temperature $T_{c}$. A useful sensor is already built within most commercial power modules. The advantage is that only the temperatures from substrate to junction have to be calculated. The thermal properties of the heatsink and cooling system are having less influence to the estimation and can be neglected.

The performance of the junction temperature estimation model is experimentally demonstrated using a 3-phase inverter with opened IGBT module. The temperatures are obtained using a high-speed infrared camera. Fig. 5 shows the junction temperature measurement for one of three phases. For the upper IGBT estimation and measurement are in a great match.
For the lower switch deviations occur. The cause can be found in the simplicity of the model, neglecting the mutual influence of the chips.

In Fig. 6 an infrared camera image of the opened IGBT module during operation is shown. Due to the arrangement of the chips on the module surface different thermal coupling factors exist. The chips in the top left corner have a strong mutual thermal influence as they are placed close to each other. As the mutual heating is not modeled in the temperature estimation, the shown inaccuracy occurs. The solve the problem a more complex thermal model can be used as described in [27].

\section{Simulation Results}

For demonstration the thermal-based FCS-MPC is applied for the permanent magnetized synchronous machine (PMSM) control. The applied field oriented control scheme of the 3phase motor is given in Fig. 8 During acceleration of an electric motor under load condition the junction temperature experiences a peak value. When steady-state is reached it will drop again. The proposed thermal-based FCS-MPC can be used to reduce the thermal stress caused by the temperature peak in the inverter. The simulation results are shown in Fig. 7.

In this demonstration the IGBT junction temperature overshoot during acceleration is compensated by temporary reduction of average switching frequency. This reduces the power losses instantaneously to reduce the junction temperature. A drawback is the increased amount of ripple in the motor current during acceleration. Expressed in terms of the optimizer, 


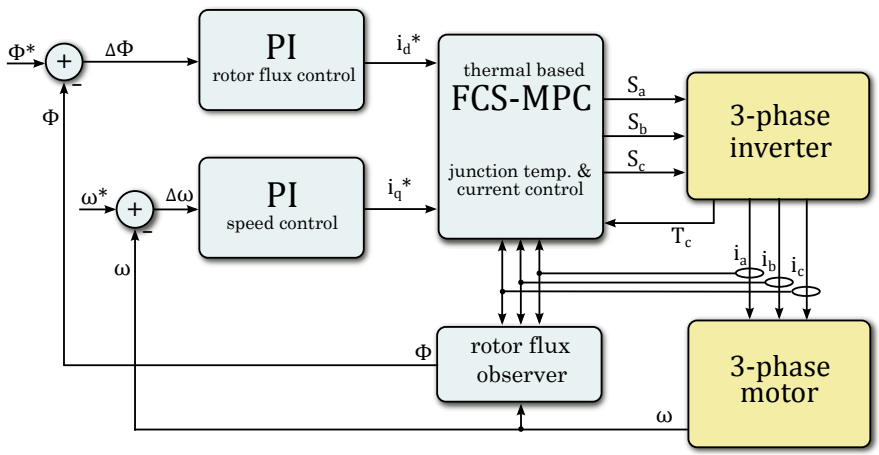

Fig. 8. Control Structure of 3-phase permanent magnetized synchronous machine. $T_{c}$ is a low-bandwidth measurement of the case temperature which is used as an input to the junction temperature estimation.

the saved module lifetime reduces more costs than the further current ripple adds.

\section{EXPERIMENTAL VALIDATION}

The experimental validation is done on a three-phase twolevel dc/ac inverter. An open module without the use of isolating gel filling is used. This allows direct temperature measurements on the chips but decreases the isolation voltage below the rated values. For power input a $10 \mathrm{~kW}$ dc power supply is used. The load is an induction machine. The control is implemented on a dSPACE DS1006 processor board.

For junction temperature measurement a high speed infrared camera is used that allows a measurement bandwidth of up to $1 \mathrm{kHz}$ for this purpose. The accuracy is rated $\pm 0.02 \mathrm{~K}$. It is used to validate the junction temperature estimation and thermal-based optimization of the FCS-MPC. A photograph of the measurement system is given in Fig. 9.

The thermal-based finite control set model predictive control is applied to optimize the thermal behavior of a three-phase IGBT module during acceleration of a $5 \mathrm{~kW}$ induction motor connected to a torque machine. Even though an asynchronous machine is used unlike the PMSM in the simulation section, the machine control scheme in Fig. 8 remains valid. The experimental results are given in Fig. 10. As a consequence to the high gradient in the junction temperatures, the FCS-MPC is selecting switching patterns that constitute less switching losses. This can be seen in the temporary decrease of the average switching frequency during acceleration. As a consequence the amplitude of the thermal overshoot is reduced.

\section{CONCLUSION}

Active thermal control uses temperature related control parameters to influence the junction temperatures online. The goal is to reduce the thermal stress in the module by smoothing the temperature variation.

The integration of active thermal control in FCS-MPC has been prepared. By using a multi-optimization towards fulfilling the application demand, reducing the thermal stress and maintaining the system efficiency, a selective use of the active thermal control can be achieved in order to maximize

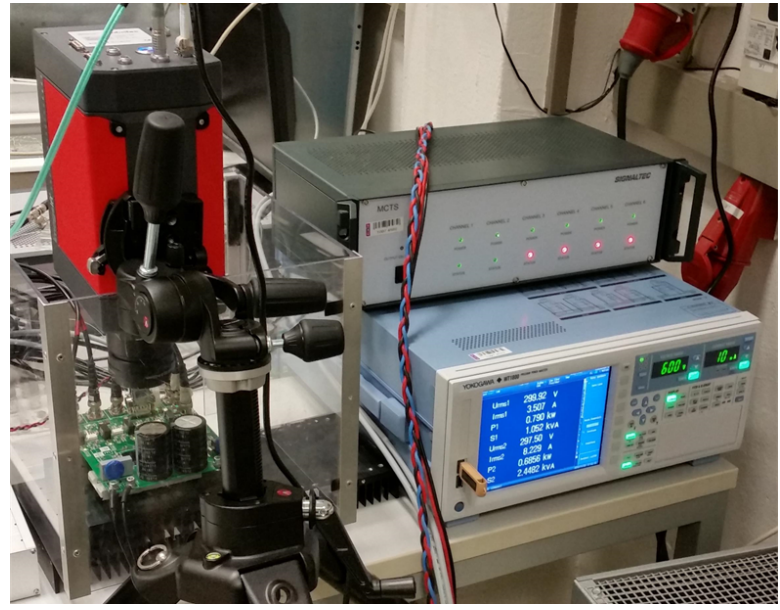

Fig. 9. High speed infrared camera experimental setup. The ir-camera is used to track the semiconductor temperatures of an opened IGBT module during operation.
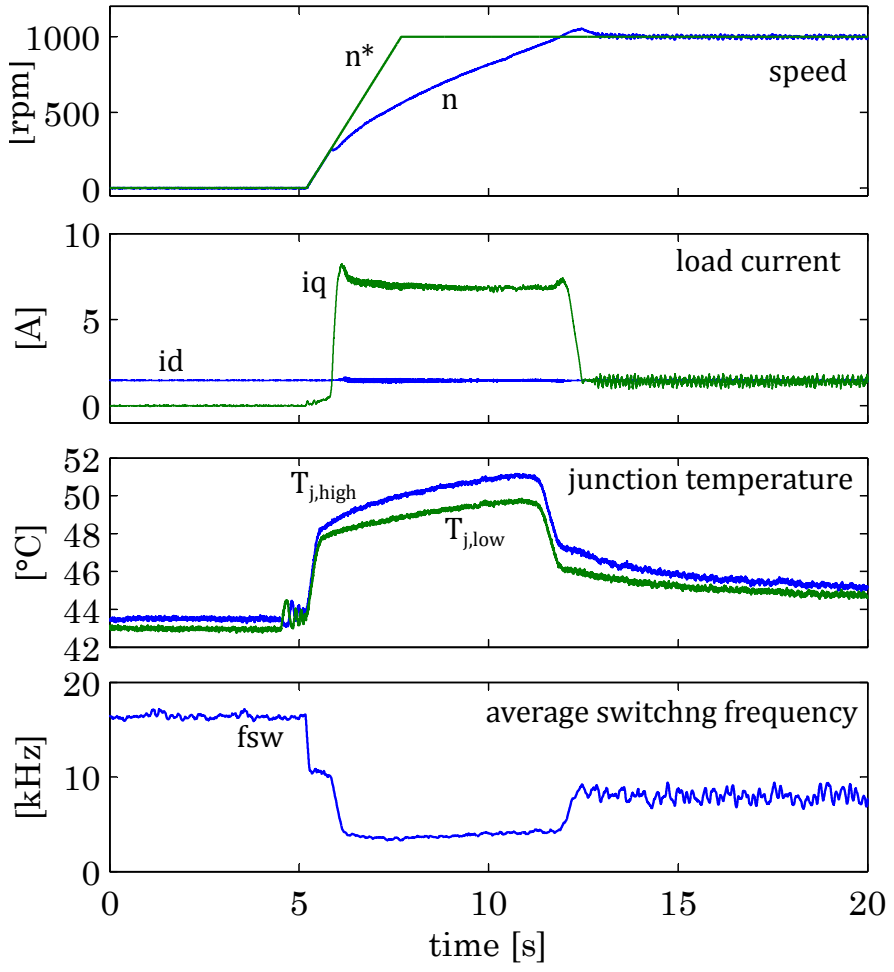

Fig. 10. Measurement of junction temperatures of an opened IGBT module during acceleration of an $5 \mathrm{kWinduction}$ motor under light mechanical load condition. DC voltage is $V_{d c}=400 \mathrm{~V}$

its benefits and minimize its drawbacks. Using the thermal optimized FCS-MPC a reduction of thermal stress in a power electronic module has been achieved during the acceleration process of electric drives without interfering other operating states. Consequentially, the thermal stress that occurs in the module can be reduced which enables an improved utilization and therefore the possibility to reduce the overrating.

A possibility for online junction temperature estimation without additional sensors has been introduced and applied 
for the FCS-MPC.

\section{ACKNOWLEDGMENT}

The research leading to these results has received funding from the Gesellschaft für Energie und Klimaschutz SchleswigHolstein $\mathrm{GmbH}$ (EKSH) doctoral studies grant and from the European Research Council under the European Unions Seventh Framework Programme (FP/2007-2013) / ERC Grant Agreement n. 616344 HEART, the Highly Efficient And reliable smart Transformer.

\section{REFERENCES}

[1] S. Kouro, M. Perez, J. Rodriguez, A. Llor, and H. Young, "Model predictive control: Mpc's role in the evolution of power electronics," Industrial Electronics Magazine, IEEE, vol. 9, no. 4, pp. 8-21, Dec 2015.

[2] H. Wang, M. Liserre, and F. Blaabjerg, "Toward reliable power electronics: Challenges, design tools, and opportunities," Industrial Electronics Magazine, IEEE, vol. 7, no. 2, pp. 17-26, June 2013.

[3] A. Wintrich, U. Nicolai, W. Tursky, and T. Reimann, "Semikron, application manual power semiconductors," Ilmenau: ISLE, 2011.

[4] A. Volke and M. Hornkamp, IGBT modules: technologies, driver and application, 2nd ed. Infineon Technologies AG, 2012.

[5] M. Schulz, "Thermal management details and their influence on the aging of power semiconductors," in Power Electronics and Applications (EPE'14-ECCE Europe), 2014 16th European Conference on. IEEE, 2014, pp. 1-6.

[6] D. Murdock, J. Torres, J. Connors, and R. Lorenz, "Active thermal control of power electronic modules," Industry Applications, IEEE Transactions on, vol. 42, no. 2, pp. 552-558, March 2006.

[7] M. Weckert and J. Roth-Stielow, "Chances and limits of a thermal control for a three-phase voltage source inverter in traction applications using permanent magnet synchronous or induction machines," in Power Electronics and Applications (EPE 2011), Proceedings of the 2011-14th European Conference on, Aug 2011, pp. 1-10.

[8] J. Lemmens, J. Driesen, and P. Vanassche, "Dynamic dc-link voltage adaptation for thermal management of traction drives," in Energy Conversion Congress and Exposition (ECCE), 2013 IEEE, Sept 2013, pp. 180-187.

[9] K. Ma, M. Liserre, and F. Blaabjerg, "Reactive power influence on the thermal cycling of multi-mw wind power inverter," Industry Applications, IEEE Transactions on, vol. 49, no. 2, pp. 922-930, March 2013.

[10] J. Zhang, H. Wang, X. Cai, S. Igarashi, Y. Li, and Z. Wang, "Thermal control method based on reactive circulating current for anticondensation of wind power converter under wind speed variations," in 2014 International Power Electronics and Application Conference and Exposition, Nov 2014, pp. 152-156.

[11] M. Bakhshizadeh, K. Ma, P. C. Loh, and F. Blaabjerg, "Indirect thermal control for improved reliability of modular multilevel converter by utilizing circulating current," in Applied Power Electronics Conference and Exposition (APEC), 2015 IEEE, March 2015, pp. 2167-2173.

[12] M. Andresen, G. Buticchi, J. Falck, M. Liserre, and O. Muehlfeld, "Active thermal management for a single-phase h-bridge inverter employing switching frequency control," in PCIM Europe 2015; International Exhibition and Conference for Power Electronics, Intelligent Motion, Renewable Energy and Energy Management; Proceedings of, May 2015, pp. 1-8.

[13] M. Ciappa, "Selected failure mechanisms of modern power modules," Microelectronics reliability, vol. 42, no. 4, pp. 653-667, 2002.

[14] M. Musallam and C. M. Johnson, "An efficient implementation of the rainflow counting algorithm for life consumption estimation," Reliability, IEEE Transactions on, vol. 61, no. 4, pp. 978-986, 2012.

[15] V. Blasko, R. Lukaszewski, and R. Sladky, "On line thermal model and thermal management strategy of a three phase voltage source inverter," in Industry Applications Conference, 1999. Thirty-Fourth IAS Annual Meeting. Conference Record of the 1999 IEEE, vol. 2, 1999, pp. 1423$1431 \mathrm{vol} .2$.

[16] M. Andresen, M. Liserre, and G. Buticchi, "Review of active thermal and lifetime control techniques for power electronic modules," in Power Electronics and Applications (EPE'14-ECCE Europe), 2014 16th European Conference on. IEEE, 2014, pp. 1-10.
[17] M. Andresen and M. Liserre, "Impact of active thermal management on power electronics design," Microelectronics Reliability, vol. 54, no. 9 pp. 1935-1939, 2014.

[18] T. Herrmann, M. Feller, J. Lutz, R. Bayerer, and T. Licht, "Power cycling induced failure mechanisms in solder layers," in Power Electronics and Applications, 2007 European Conference on, Sept 2007, pp. 1-7.

[19] J. Lemmens, P. Vanassche, and J. Driesen, "Optimal control of traction motor drives under electrothermal constraints," Emerging and Selected Topics in Power Electronics, IEEE Journal of, vol. 2, no. 2, pp. 249-263, June 2014.

[20] J. Holtz and S. Stadtfeld, "A predictive controller for the stator current vector of ac machines fed from a switched voltage source," in JIEE IPEC-Tokyo Conf, 1983, pp. 1665-1675.

[21] J. Rodriguez, M. Kazmierkowski, J. Espinoza, P. Zanchetta, H. AbuRub, H. Young, and C. Rojas, "State of the art of finite control set model predictive control in power electronics," Industrial Informatics, IEEE Transactions on, vol. 9, no. 2, pp. 1003-1016, May 2013.

[22] E. F. Camacho and C. B. Alba, Model predictive control. Springer Science \& Business Media, 2013.

[23] L. Wang, Model predictive control system design and implementation using MATLAB. Springer Science \& Business Media, 2009.

[24] S. Kouro, P. Cortes, R. Vargas, U. Ammann, and J. Rodriguez, "Model predictive control: A simple and powerful method to control power converters," Industrial Electronics, IEEE Transactions on, vol. 56, no. 6, pp. 1826-1838, June 2009.

[25] M. Preindl, E. Schaltz, and P. Thogersen, "Switching frequency reduction using model predictive direct current control for high-power voltage source inverters," IEEE Transactions on Industrial Electronics, vol. 58, no. 7, pp. 2826-2835, July 2011.

[26] J. Falck, M. Andresen, and M. Liserre, "Active thermal control of igbt power electronic converters," in Industrial Electronics Society, IECON 2015-41st Annual Conference of the IEEE. IEEE, 2015, pp. $000001-$ 000006.

[27] H. Wang, A. M. Khambadkone, and X. Yu, "Control of parallel connected power converters for low voltage microgrid part ii: Dynamic electrothermal modeling," IEEE Transactions on Power Electronics, vol. 25, no. 12, pp. 2971-2980, Dec 2010. 